\title{
ВЫБОР УЧЕБНОГО МАТЕРИАЛА ДЛЯ УГЛУБЛЕННОГО ИЗУЧЕНИЯ ДИСЦИПЛИНЫ «НАЧЕРТАТЕЛЬНАЯ ГЕОМЕТРИЯ»
}

\section{SELECTION OF EDUCATIONAL MATERIAL FOR IN-DEPTH STUDY OF THE DISCIPLINE "DESCRIPTIVE GEOMETRY"}

\section{E. Stepura}

Summary: The author tried to formulate a number of methodically-based criteria for selecting educational material for the work of scientific circles on descriptive geometry. The purpose of this type of training is to provide a high level of professional competence in the study of the disciplines "Descriptive geometry", "Descriptive geometry and engineering graphics», "Engineering and computer graphics», etc., as well as to create a theoretical basis for studying related disciplines and conducting research in senior courses. Within the framework of the scientific circle, the development and testing of methods and techniques for working with gifted students is carried out, the principle of an individual approach to learning is implemented, effective ways of managing students independent work are worked out. The author offers a classification of educational tasks based on the mental operations necessary to solve them, and also highlights those techniques of operating with spatial images that cause difficulties for most students and require additional work to master them. of developing students' spatial thinking skills. We used the experience of leading a scientific circle of the descriptive geometry on part (for the purpose of preparing the University team for the regional and all-Russian rounds of the student Olympiad) and reviewing students reports circle members. Observations and individual interviews were conducted. The author suggests topics for group classes that correspond to the main stages of developing students 'spatial thinking skills. Taking into account the regularities of the internal side of the learning process can significantly improve the effectiveness of teaching the discipline.

Keywords: independent work of students, educational material on descriptive geometry, professional competence, individual approach to training.

\author{
Степура Екатерина Анатольевна \\ Московский государственный строительный \\ университет, г. Москва \\ StepuraEA@mgsu.ru
}

Аннотация: Автор попытался сформулировать ряд методически-обоснованных критериев подбора учебного материала для работы научных кружков по начертательной геометрии. Целью занятий такого рода является высокий уровень овладения профессиональными компетенциями при изучении дисциплин «Начертательная геометрия», «Начертательная геометрия и инженерная графика», «Инженерная и компьютерная графика» и др., а также создание теоретического базиса для изучения смежных дисциплин и проведения научных исследований на старших курсах. В рамках занятий научного кружка, осуществляются разработка и апробация методов и приемов работы с одаренными студентами, реализуется принцип индивидуального подхода в обучении, отрабатываются эффективные способы руководства самостоятельной работой студентов. Автор предлагает классификацию учебных задач, построенную с учетом мыслительных операций, необходимых для их решения, а также выделяет те приемы оперирования пространственными образами, которые вызывают трудности у большинства студентов и требуют дополнительной работы для овладения ими. Использован опыт руководства научным кружком по начертательной геометрии с целью подготовки команды вуза к региональному и Всероссийскому турам студенческой олимпиады, а также рецензирования исследовательских работ студентов. Проводились наблюдения и индивидуальные беседы. Автор предлагает тематику кружковых занятий, соответствующую основным этапам развития навыков пространственного мышления обучаемых. Учет закономерностей внутренней стороны процесса обучения позволяет значительно повысить эффективность преподавания дисциплины.

Ключевые слова: самостоятельная работа студентов, учебный материал по начертательной геометрии, профессиональные компетенции, индивидуальный подход в обучении.
$\mathrm{T}$ ак как базовые знания по дисциплине «Начертательная геометрия» (и дисциплинам, в которые она входит как раздел) студенты получают в ходе аудиторных занятий, то учебный материал для углубленного изучения данного предмета отличается, прежде всего, своей развивающей направленностью. Цель кружковых занятий с одаренными студентами -высокий уровень овладения профессиональными компетенциями, который предполагает самостоятельное решение нестандартных, творческих задач на базе высоко развитого пространственного мышления. Отметим, что иногда начертательную геометрию объявляют устаревшей дис- циплиной, которую можно полностью заменить компьютерной графикой и моделированием. При рассмотрении психолого-педагогических основ преподавания предмета, вскрывается ошибочность такого рода суждений. В начертательной геометрии (как учебной дисциплине), трудами нескольких поколений ученых - педагогов, сложилась хорошо работающая методика поэтапного формирования навыков пространственного мышления, крайне необходимых будущему инженеру в его профессиональной деятельности: начиная с чтения чертежей и заканчивая расчетами и проектированием уникальных конструкций. К сожалению, в настоящее время имен- 
но эта сторона мышления у выпускников школ развита крайне слабо, что требует от педагога-практика напряженной дополнительной работы.

В рамках цифровизации процесса обучения, мы все шире применяем в преподавании современные технологии, но при этом отсутствие понимания психологических основ процесса обучения, создает соблазн заменить теоретическое (развивающее) содержание учебного предмета - внешней инструментальной формой, с помощью которой оно преподносится. Заменить процесс формирования умений и навыков работы с чертежом - заучиванием стандартных алгоритмов и ГОСТОВ, а понимание - зубрежкой типовых задач и программ.

Подбирая учебный материал для занятий научного кружка, автор классифицировал учебные задачи и упражнения в соответствии с определенным типом оперирования пространственным образом геометрического объекта, который наблюдается во внутренней мыслительной деятельности при поиске решения. Необходимо отметить, что любая задача, решаемая на основе проекционных изображений (чертежа), вначале мысленно переводится субъектом в пространственный образ. Затем происходит оперирование этим образом с целью нахождения идеи решения задачи (в пространстве) и создается представление о плане решения - только после этого решение может быть реализовано на чертеже с помощью того или другого инструмента и способа изображения.

К^ассификашия учебных задач по типу оперирования пространственным образом геометрического объекта.

1. Изменение структуры пространственного образа. Задачи на построение сечений и линий пересечения геометрических тел.

2. Изменение положения пространственного образа объекта по отношению к системе отсчета или другим объектам.

Метрические и позиционные задачи, решаемые с применением способов преобразования проекций.

3. Изменение положения пространственного образа с изменением его структуры.

Задачи по конструированию геометрических объектов с заданными параметрами и свойствами.

Наибольшие трудности у обучающихся вызывает выполнение упражнений, указанных в пункте 3. Но именно решение такого рода задач является показателем достаточно высокого уровня развития пространственного воображения, позволяющего будущему специалисту вполне самостоятельно и творчески заниматься моделированием и расчетами поверхностей, полноценно ис- пользуя для этого возможности искусственного интеллекта.

С учетом поставленной нами цели: соотнесения этапов учебного процесса и выбора учебного материала (на каждом этапе) с основными периодами формирования необходимых приемов умственной деятельности студентов, была составлена тематика занятий кружка.

Необходимо отметить, что учебный материал, используемый в ходе работы кружка, был использован студентами при выполнении небольших собственных исследований. Результаты этих исследований представлялись в виде докладов на ежегодной студенческой конференции «Дни студенческой науки» в НИУ МГСУ, а также применялись студентами при самостоятельной подготовке к олимпиадам по начертательной геометрии [3]. Ниже приводятся ссылки на эти доклады.

График работы кружка был разбит на этапы; длительность каждого этапа варьировалась в зависимости от уровня базовых знаний участников кружка и имеющихся ресурсов времени. Предусматривались семинары, задания для самостоятельной работы студентов; проводилось обсуждение решений, предложенных участниками кружка.

1 этап. Изучение способов задания и свойств поверхностей. Рассматривались правильные многогранники; поверхности с плоскостью параллелизма; эллиптические (наклонные) конус и цилиндр; однополостный гиперболоид вращения, открытый и закрытый тор. Решались задачи построения сечений этих поверхностей плоскостями общего и частного положений, а также проведения касательных плоскостей к кривым поверхностям [2]. Студенты подводились к выводу: исследование ряда последовательных сечений поверхности позволяет лучше представить форму, а также изучить ее геометрические свойства [1].

При решении задач построения линий взаимопересечения поверхностей, расширялись знания о методах решения (метод «пучка качающихся плоскостей», метод сфер в разных вариантах, теорема «о двойном соприкосновении» при построении круговых сечений и др.) [5]. Особое внимание уделялось тому, чтобы мысленные представления, создававшиеся в ходе решения задач, отличались динамизмом: «качающиеся плоскости», «пульсирующие» сферы и т.д.

На данном этапе велась целенаправленная работа по расширению и совершенствованию представлений о пространственной структуре различных поверхностей на основе изучения их проекционных изображений. Отрабатывалась также способность представлять последовательные изменения формы объекта. Шло форми- 
рование рациональных приемов перехода от плоских проекционных изображений поверхностей к их пространственным образам, переход к «свернутым» формам и автоматизмам этого процесса. Накопленный опыт исследования геометрии поверхностей, стал фундаментом для более эффективных мысленных действий в процессе чтения чертежа и построения изображений [7].

2 этап. Применение способов преобразования проекций при решении практических задач с поверхностями.

На втором этапе было сформировано целостное представление о теоретических основах способов преобразования проекций, в частности - о закономерностях и признаках отображения на чертеже изменения положения системы отсчета (плоскостей проекций) относительно неподвижного объекта; а также перемены положения геометрического объекта относительно неподвижной системы плоскостей проекций. Рассматривались различные варианты способа вращения, способ замены плоскостей проекций, способ косоугольного проецирования. Была очерчена область применения этих способов к решению определенного круга метрических и позиционных задач.

На базе уже достаточно хорошо сформированных приемов работы с изображениями и пространственными образами поверхностей, отрабатывались способы геометрически обоснованного изменения проекционных изображений пространственного объекта, отображающих его мысленное перемещение в пространстве. Подготовительными операциями были: представить тело с разных ракурсов, выбрать наиболее удачный ракурс и геометрически правильно описать его, выявить закономерности, соответствующие изменению положения объекта, доказать эффективность выбранного способа решения. Широко использовались наброски, эскизы, рисунки. Развитие приемов мышления велось в направлении все большего динамизма и свободы в создании пространственных образов и оперирования ими при решении различных задач.

3 этап. Моделирование геометрических объектов с заданными характеристиками.

Задачи такого рода предлагаются на этапах Всероссийской олимпиады по начертательной геометрии, инженерной и компьютерной графике. Студенты, успешно выступавшие и побеждавшие на Олимпиаде, показали высокий уровень владения приемами работы с пространственными образами. Можно говорить о синергии первых двух типов оперирования пространственным образом, свидетельствующей о переходе на качественно новый, несущий отпечаток индивидуальности, этап развития пространственного мышления. Необходимо заметить, что память, словесно-логическое и простран- ственное мышление тесно связаны, развитие одной из сторон мышления всегда способствует совершенствованию других, что постоянно наблюдается при решении геометрических задач.

На этом этапе использовались задачи на построение геометрических мест, к сожалению, почти исчезнувшие из современной учебной литературы. На актуальность такого рода упражнений обращали внимание известные специалисты: А.И. Добряков [5, с.211], В.О. Гордон [4, с.375], А.К. Рудаев [8, с.716], выделяя эти задачи в особый раздел.

При построении различных геометрических мест, необходимо мысленно создать поверхность с заданными параметрами, при этом студент должен вначале самостоятельно сформулировать свойства такого объекта, исходя из условия задачи. Далее строятся сечения полученной поверхности или решается задача пересечения двух поверхностей. Часто в ходе решения используются различные способы преобразования проекций [6]. Способность самостоятельно находить решение такого рода задач и геометрически грамотно отображать его на проекционном чертеже свидетельствует о достигнутом высоком уровне развития компетенций.

\section{Выводы}

Качество учебного материала, используемого в работе научного кружка, не может сводиться лишь к расширению объема информации по темам программы. Автор попытался, с помощью целенаправленного выбора учебных задач (по критерию: тип мыслительных операций в процессе работы над решением), реализовать принцип развивающего обучения; на этой основе сформировать у студентов качества, необходимые для дальнейшей самостоятельной творческой работы, как в области начертательной геометрии, так и смежных дисциплин на старших курсах. Предложенная тематика и виды учебных задач направлены на формирование определенных приемов пространственного мышления, являющихся базой для высокого уровня развития профессиональных компетенций будущего инженера. Подтверждением правильности такого подхода служат хорошие результаты выступлений команды МГСУ на региональных (призовые места ежегодно) и Всероссийском турах студенческой олимпиады по начертательной геометрии, инженерной и компьютерной графике, (студенты МГСУ Зонтов Р.А., Мокин Н.А.- победители Олимпиады). Стабильно отмечается высокая успеваемость и активная исследовательская работа выпускников научного кружка «Начертательная геометрия» на старших курсах. Также, предложенный вариант построения программы занятий может быть использован при разработке методик и приемов внедрения цифровых технологий в учебный процесс по графическим дисциплинам. 


\section{ЛИТЕРАТУРА}

1. Арташина А.Э. Бегущая спираль: геометрия и конструкция. Дни студенческой науки./ Сборник докладов научно-технической конференции по итогам НИРС института строительства и архитектуры. -Москва. МГСУ. 2017, с.129-132.

2. Брызгалин В.В. Построение касательной плоскости к трем сферам./ Сборник докладов научно-технической конференции по итогам НИРС института строительства и архитектуры. - Москва. МГСУ. 2014, с.119-121.

3. Вышнепольский В.И. Московские городские олимпиады по инженерной графике./Геометрия и графика. Сборник научных трудов. - Москва. МИТХТ им. М.В. Ломоносова. 2011, с.171-180.

4. Гордон В.О., Семенцов-Огиевский М.А. Курс начертательной геометрии. Гос. изд-во технико-теоретической лит-ры. Москва.1956, 404 с.

5. Добряков А.И. Курс начертательной геометрии. Гос. изд-во литературы по строительству и архитектуре, - Москва. 1952, 487 с.

6. Мокин Н.А. Задачи на применение геометрических мест./ Промышленное и гражданское строительство в современных условиях. Материалы международной научно - технической конференции студентов.- Москва. МГСУ, 2011, с. 470-472.

7. Мокин Н.А., Попов А.Г. Несобственные элементы при центральном проектировании./ Сборник докладов научно-технической конференции по итогам НИРС института строительства и архитектуры.- Москва. МГСУ. 2013, с. 191-193.

8. Рудаев А.К. Сборник задач по начертательной геометрии. ОГИЗ ГОСТЕХИЗДАТ. - Москва, 1948, 344 с.

(c) Степура Екатерина Анатольевна (StepuraEA@mgsu.ru).

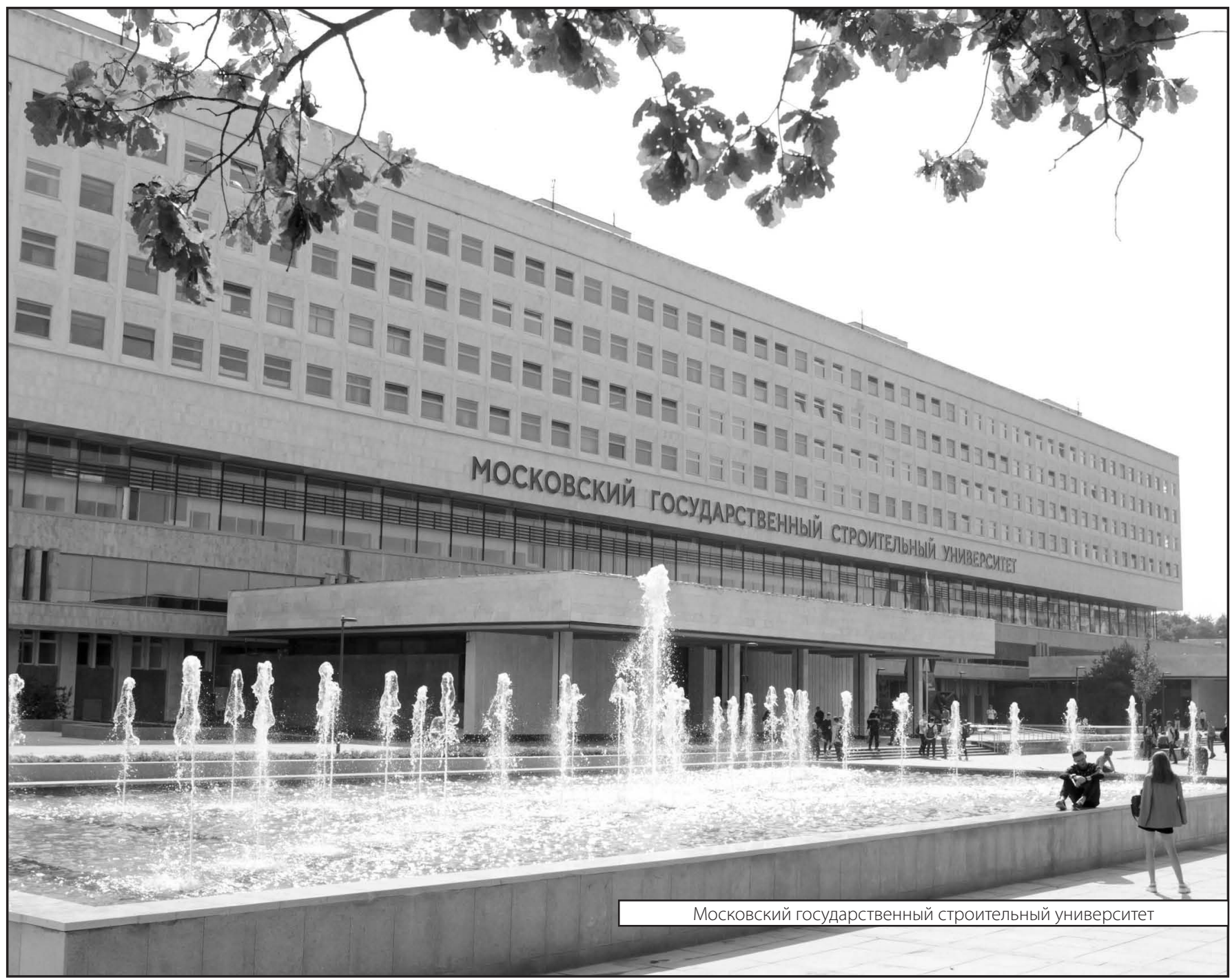

